\title{
DEVELOPMENT AND CHARACTERIZATION OF CREAM CONTAINING NANOSTRUCTURED LIPID CARRIERS FOR HAIR SPLIT-END REPAIRING
}

\author{
TASHATAI PRASERTPOL ${ }^{a, b}$, WAREE TIYABOONCHAI $I^{a, b, c^{*}}$
}

aFaculty of Pharmaceutical Sciences, Naresuan University, Phitsanulok, Thailand, bThe Center of Excellence for Innovation in Chemistry (PERCH-CIC), Commission on High Education, Ministry of Education, Thailand, cThe Center of Excellence in Medical Biotechnology, Naresuan University, Phitsanulok, Thailand

Email: wareet@nu.ac.th

Received: 29 Apr 2019, Revised and Accepted: 01 Jun 2019

\section{ABSTRACT}

Objective: This study aimed to develop the nanostructured lipid carriers (NLCs) for repairing the hair split-end and to compare the influence of NLCs charges on hair binding effects.

Methods: NLCs was prepared by a high-pressure homogenization technique. The difference solid lipids were selected to obtain the negatively charged NLCs (N-NLCs) and positively charged NLCs (P-NLCs). The physical characterizations of both NLCs were examined. The NLCs cream was prepared by mixing NLCs into a cream base. Then, the hair split-end binding effects of the NLCs cream were assessed. The physical stability of both NLCs were investigated by kept at room temperature for 6 mo.

Results: The N-NLCs and P-NLCs were successfully prepared with a zeta potential of $-44.4 \mathrm{mV}$ and $32.3 \mathrm{mV}$, respectively. They were a spherical shape with a similar mean size of $\sim 150-160 \mathrm{~nm}$. The hair split-end evaluation showed that both NLCs illustrated similar hair split-end binding time of more than $3 \mathrm{~d}$, suggesting no extra benefit from the positively charged of P-NLCs. On the contrary, the cream base and commercial product showed a short binding time of $<8 \mathrm{~h}$. Furthermore, after 6-mo storage, N-NLCs demonstrated good physical stability without particle aggregation, while P-NLCs exhibited a phase separation.

Conclusion: The developed NLCs is a novel alternative for the leave-on product, which would be highly benefits for hair split-end repairing.

Keywords: Nanostructured lipid carriers, Human hair, Split-end, Leave-on product

(C) 2019 The Authors. Published by Innovare Academic Sciences Pvt Ltd. This is an open access article under the CC BY license (http://creativecommons.org/licenses/by/4.0/) DOI: http://dx.doi.org/10.22159/ijap.2019v11i4.33836

\section{INTRODUCTION}

In general, most hair damages occur inevitably by chemical treatments such as waving, relaxing, bleaching, and coloring process leading to hair fuzzy. These cosmetic practices could damage the hair protein structure from the outermost layer as cuticle to the cortex, resulting in the loss of hair mechanical strength and formation of split-ends [1-3]. Furthermore, the mechanical process of heat styling and combing could lead to more severe longitudinal split-end, which resulting in hair breakage [4]. These effects are undesirable because the good appearance of hair is related to selfconfidence and personality for the human being. In fact, the hair damage could be retarded by repairing the hair split-end. Thus, the use of hair care product for temporarily repairing the hair split-end is widely practiced [5].

Accordingly, a wide variety of hair product (leave-on) have been developed with aimed to repair hair split-end. The most common active substances have been used including oils, silicone, and polymer [6-8]. These substances are designed to deposit on the outermost cuticle layers, covering the hair damages and binding the split-end fibers back together with long-lasting effect and safe. Unfortunately, they have been notoriously limited [9]. For instance, the positively charged polymers and silicones were developed to provide a higher affinity to the negatively charged hair fibers. However, they tend to accumulate on hair, which may cause irritation on head skin. To avoid those polymers and silicone, the currently leave-on product is usually used natural oil. Nevertheless, they exhibited low effectiveness for hair split-end binding effect. Thus, to fulfill leave-on product for hair splitend repairing, a strategy for improving its biocompatibility and adhesion ability is still a challenge task.

Nanostructured lipid carriers (NLCs) has been proposed as a novel potential alternative to overcome those limitations [10]. NLCs have been developed to combine the advantages of other colloidal carriers but without their disadvantages [11-13]. For example, NLCs can be prepared using physiological lipids similar to nanoemulsions and liposomes, thus providing less irritation problems compared to polymeric nanoparticles. Nevertheless, NLCs show better physical stability than nanoemulsions and liposomes by remaining in a solid state at room and body temperature. Additionally, NLCs possess a good adhesive ability due to its small particle in nano range [14, 15]. These advantages could be a strategy to repair the hair split-end.

Therefore, in this study, NLCs were prepared by high-pressure homogenization technique. We further developed the negative and positive charged NLCs to compare the influence of electrostatic charges on hair split-end binding effect. The physicochemical properties of NLCs in term of particle size, zeta potential, and morphology were then investigated. In addition, the NLCs were prepared as a leave-on product by mixing NLCs into the cream base and then the effectiveness of NLCs cream was also examined on hair split-end fibers.

\section{MATERIALS AND METHODS}

\section{Materials}

EmulmetikVR900 (Soybean phospholipids, E900) was purchased from Lucas Meyer Cosmetic (Champlan, France). Glyceryl monostearate, stearic acid, myristic acid, and propylene glycol were purchased from Namsiang trading (Bangkok, Thailand). Span 80 and Tween 80 were purchased from Phitsanuchemical (Phitsanulok, Thailand). Stearyl amine was purchased from Sigma-Aldrich Co. (Missouri, USA). All other chemicals and solvents were of analytical grade. Regenerated cellulose membrane, diameter $76 \mathrm{~mm}$, with a molecular weight cut off (MWCO) $100 \mathrm{kDa}$ (MiliporeVR YM100) was purchased from Millipore Corporation (MA, USA). Damaged hair was purchased from Tokyo beauty establishment (Phitsanulok, Thailand), each hair samples was collected from one single donor.

\section{Methods}

\section{Preparation of NLCs formulations}

Negatively charged NLCs (N-NLCs) and positively charged NLCs (PNLCs) were prepared by a high-pressure homogenization technique 
[16]. Briefly, both N-NLCs and P-NLCs were prepared with the same formulation base as follows: $4 \%(\mathrm{w} / \mathrm{w})$ of Span $80,3 \%(\mathrm{w} / \mathrm{w})$ of Tween 80, $0.7 \%(\mathrm{w} / \mathrm{w})$ of E900, $0.5 \%(\mathrm{w} / \mathrm{w})$ of glyceryl monostearate. In addition, to obtain the negative or positive charged NLCs, the different types of anionic or cationic lipids at constant total lipid concentration $(1 \% \mathrm{w} / \mathrm{w})$ were added into formulations base. The N-NLCs was prepared with $0.5 \%(\mathrm{w} / \mathrm{w})$ of stearic acid and 0.5 $\%(\mathrm{w} / \mathrm{w})$ of myristic acid. Meanwhile, $1 \%(\mathrm{w} / \mathrm{w})$ of stearyl amine was chosen to achieve the P-NLCs. Then, the oil phase and water phase were separately heated to $80^{\circ} \mathrm{C}$. The water phase was further poured into the oil phase and homogenized using a high shear homogenizer (IKA, Staufen, Germany) at $8000 \mathrm{rpm}$ for $2 \mathrm{~min}$.

The resulting hot pre-emulsion of N-NLCs and P-NLCs were processed through a high-pressure homogenizer (M-110P, Microfluidics, Massachusetts, USA) at 1000 bar for 5 and 7 cycles respectively. The obtained nanoemulsions were then cooled down to ambient temperature for resolidification the lipid and forming the NLCs. Finally, both N-NLCs and P-NLCs were washed three times with deionized water (DI) using an ultrafiltration cell fitted with an MWCO 100 kDa membrane (Millipore, Massachusetts, USA).

\section{Physical characterization of N-NLCs and P-NLCs}

The mean particle size and polydispersity index (PI) were determined by dynamic light scattering (DLS) with a ZetaPALS® analyzer (Brookhaven Instrument Corporation, New York, USA). This instrument was equipped with a $35 \mathrm{~mW}$ HeNe laser diode operating at $632.8 \mathrm{~nm}$ and a BI-200SM Goniometer connected to a BI-9010AT digital correlator. All samples were dispersed in DI water and run for 10 measurement cycles. The mean particle size and PI values were obtained by the auto measuring mode at a fixed angle of $90^{\circ}$. The particle size data were analyzed using the hydrodynamic diameter. The measurement was performed in triplicate.

The zeta potential was determined by measuring the particle electrophoretic mobility, using the ZetaPAL® analyzer. The measurement was then carried out at an angle of $14.8^{\circ}$ to the incident light. All samples were dispersed in DI water and run for 10 measurement cycles. The zeta potential was calculated based on the Smoluchowski equation.

The morphology of N-NLCs and P-NLCs were examined using a transmission electron microscope (TEM, Philips, Oregon, USA). In the preparation process, $20 \mu \mathrm{l}$ of each sample was deposited on a carbon-coated 300 mesh copper grid. Then, $10 \mu \mathrm{l}$ of $0.5 \%(\mathrm{w} / \mathrm{v})$ uranyl acetate in ethanol was dropped onto the grid. The excessive solvent was removed by Whatman no. 1 filter paper and allowed to air-dry at room temperature. The dried samples were kept in a desiccator for further observation by TEM.

\section{Preparation of a model cream base and NLCs cream}

The model cream base was prepared by a beaker method. The oil phase and the water phase were heated to $70-75{ }^{\circ} \mathrm{C}$. The water phase was added into the oil phase, stirring until cooling down to room temperature. To obtain NLCs cream, $70 \%(w / w)$ of NLCs was dispersed into a model cream base and mixed. In addition, the cream base without NLCs dispersions was used as a control.

\section{Evaluation of NLCs cream on hair split-ends repairing}

Damaged hair was collected from one single donor. Then, the damaged hair tress was washed with nonionic shampoo and rinsed with water. After air-drying at room temperature, 45 fibers of hair split-end were randomly chosen with a length of $\sim 7 \mathrm{~cm}$. Each hair fiber was treated with a drop of hair split-end repairing products ( 9 fibers/product): $\mathrm{N}$ NLCs cream, P-NLCs cream, commercial A, commercial B and cream base. Before and after treatment, the same surface area of each hair fiber was observed under a light microscope (Motic BA300, New York Company, China) [5]. A possible change of samples was observed at 12 hourly intervals/day for 3 consecutive days.

\section{Physical stability of NLCs dispersions}

N-NLCs and P-NLCs dispersions were stored at room temperature in a well-closed container. An aliquot of each sample was taken at intervals of 3 and 6 mo to investigate the mean particle size and zeta potential. Each sample was carried out in triplicate.

\section{Statistical analysis}

The results were expressed using the mean \pm standard deviation (SD). Differences between the groups were compared by one-way ANOVA, which followed by multiple comparison post hoc test. The results were considered to be the statistically significant difference at $P$ value $<0.05$.

\section{RESULTS}

\section{Physical characterization of NLCs dispersions}

In this study, N-NLCs and P-NLCs were successfully prepared to obtain the negatively and positively charged particles respectively. P-NLCs containing stearyl amine illustrated the positively charged with a zeta potential of $32.3 \mathrm{mV}$, table 1 . In the meantime, preparing N-NLCs with stearic acid and myristic acid showed a negatively charged with a zeta potential of $-44.4 \mathrm{mV}$. However, they showed a slightly different in mean particle size, as shown in table 1. The particle size of P-NLCs $(169 \mathrm{~nm})$ was slightly larger than N-NLCs $(156 \mathrm{~nm})$ and the PI value of both formulations was less than 0.3 , indicating a narrow size distribution. Furthermore, the TEM micrographs of N-NLCs and P-NLCs exhibited spherical shape and showed a similar particle size to those obtained by DLS, as shown in fig. 1.

Table 1: Mean particle size, polydispersity index and zeta potential of N-NLCs and P-NLCs

\begin{tabular}{llll}
\hline Formulation & Mean particle size (nm) \pm SD & Polydispersity index \pm SD & Zeta potential (mV) \pm SD \\
\hline N-NLCs & $156 \pm 1$ & $0.247 \pm 0.018$ & $-44.4 \pm 4.0$ \\
P-NLCs & $169 \pm 2$ & $0.262 \pm 0.023$ & $+32.3 \pm 1.0$ \\
\hline
\end{tabular}

Note: $\mathrm{SD}=$ standard deviation for $\mathrm{n}=3$

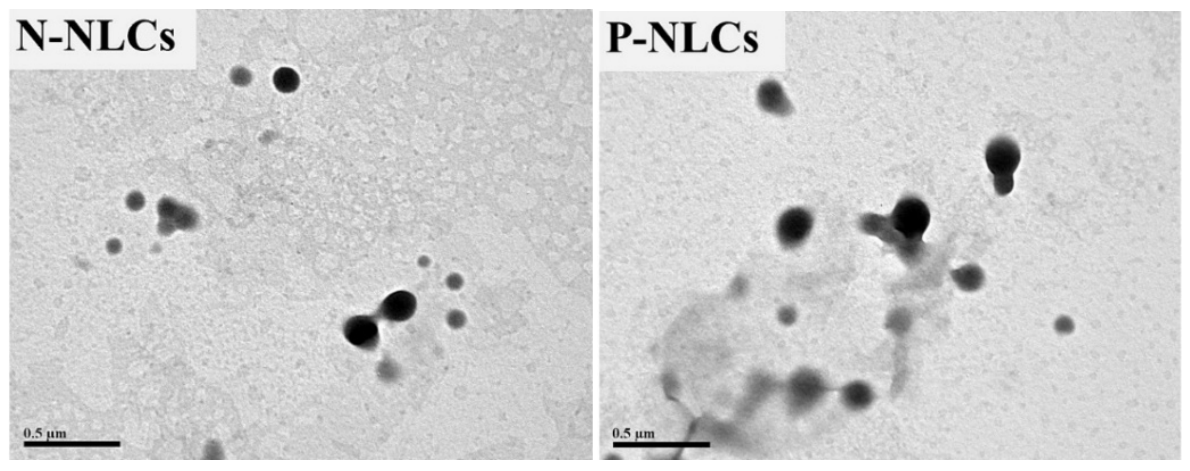

Fig. 1: TEM micrographs of N-NLCs and P-NLCs 


\section{Evaluation of NLCs on hair split-end repairing}

The effectiveness of the developed NLCs on hair split-end repairing was examined using a light microscope, under $40 \mathrm{x}$ and $100 \mathrm{x}$ magnification. In this study, the hair split-end were randomly chosen from the damaged hair tress and treated with the difference products as follows: N-NLCs cream, P-NLCs cream, commercial A, and commercial B products. The cream base was also applied as a control. The morphological characteristics of a split-end point were categorized into 2 stages including initial split-end and deep split-end. Initial split end indicates the repaired fiber initially splitting again after combined together, while deep split end indicates the severe fiber splitting following initial fiber splitting. In addition, the time required for each split-endpoint was determined to compare the binding ability of each sample.

\begin{tabular}{|c|c|c|c|}
\hline \multirow{2}{*}{ Formulation } & \multirow{2}{*}{ Hair split-end } & \multicolumn{2}{|c|}{ After treatment } \\
\hline & & 40x magnification & $100 x$ magnification \\
\hline \multicolumn{4}{|l|}{ N-NLCs cream } \\
\hline \multicolumn{4}{|l|}{ P-NLCs cream } \\
\hline \multicolumn{4}{|l|}{ Cream base } \\
\hline \multicolumn{4}{|l|}{ Commercial A } \\
\hline & 100 & $100 \mathrm{\mu m}$ & $100 \mu \mathrm{\mu m}$ \\
\hline Commercial B & & & \\
\hline & & $\stackrel{100 \mathrm{pm}}{=}$ & \\
\hline
\end{tabular}

Fig. 2: The micrographs of the hair split-end before and after treated with difference samples at 40x and 100x magnification

As shown in fig. 2, after treatment with samples, all products were able to combine the hair-splitting back together. However, the repaired hair could split again, depending on the adhesion effect and film formation ability of each formulation. The hair treated with a cream base, commercial A and B products demonstrated an initial split-end at $2.5,3.2$, and $4.1 \mathrm{~h}$ respectively, and they continued to split until reaching the deep split-end at $4.4,6.4$, and $8.4 \mathrm{~h}$ respectively. On the contrary, the hair treated with $\mathrm{NLC}_{\mathrm{S}}$ creams showed the greatest hair repairing effect. As evidence from fig. 3, the hair treated with N-NLCs and P-NLCs creams demonstrated initial split-end at $\sim 40$ and $\sim 42 \mathrm{~h}$ respectively with no statistically significant difference. Moreover, they were able to pull a hair splitend fiber back together for a long time period before indicating deep split-end of more than $72 \mathrm{~h}$.

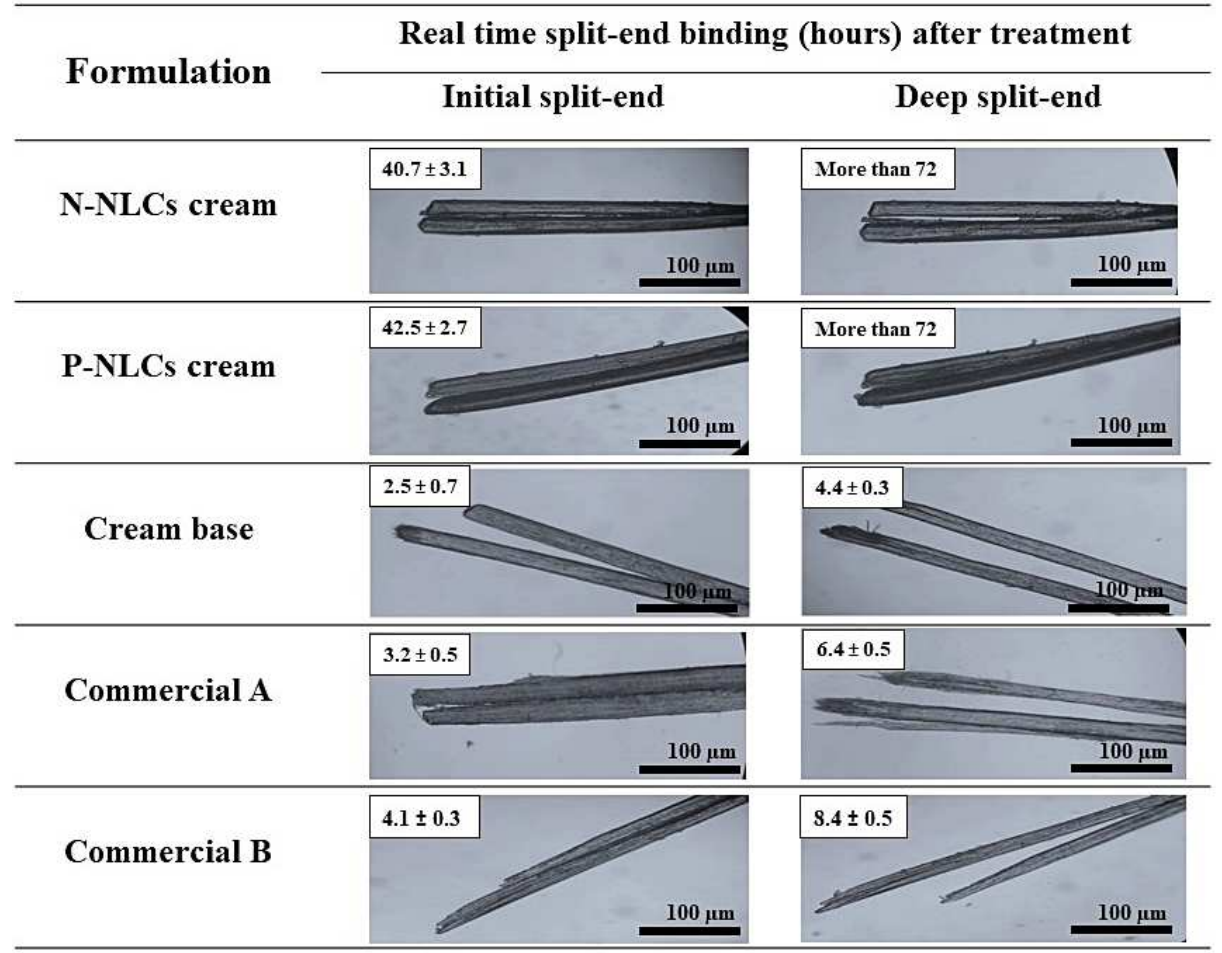

Fig. 3: The micrograph at 100x magnification of the split-end binding duration ( $\mathrm{SD}=$ standard deviation for $\mathrm{n}=9$ ) 


\section{Physical stability NLCs dispersions}

After 6 mo-storage at room temperature, the N-NLCs revealed a great physical stability, table 2 . The mean particle size was practically unchanged of $\sim 150-160 \mathrm{~nm}$ and zeta potential showed no significant difference compared with a freshly prepared. In contrast, P-NLCs demonstrated a larger particle size of $\sim 343 \mathrm{~nm}$ after 3-mo storage and decreased in zeta potential from 32 to 23 $\mathrm{mV}$. Furthermore, the phase separation was observed after 6-mo storage.

Table 2: Physical characterization of N-NLCs and P-NLCs after 6-mo storage at room temperature

\begin{tabular}{lllll}
\hline Formulation & Storages time & Mean particle size (nm) \pm SD & Polydispersity index \pm SD & Zeta potential (mV) \pm SD \\
\hline \multirow{3}{*}{ N-NLCs } & Initial & $156 \pm 1^{\mathrm{a}}$ & $0.247 \pm 0.018$ & $-44.1 \pm 1.4^{\mathrm{c}}$ \\
& $3 \mathrm{mo}$ & $160 \pm 1^{\mathrm{a}}$ & $0.274 \pm 0.011$ & $-45.9 \pm 1.1^{\mathrm{c}}$ \\
& $6 \mathrm{mo}$ & $163 \pm 2^{\mathrm{a}}$ & $0.264 \pm 0.009$ & $-44.3 \pm 1.1^{\mathrm{c}}$ \\
P-NLCs & Initial & $169 \pm 2^{\mathrm{a}}$ & $0.262 \pm 0.023$ & $+32.3 \pm 1.6^{\mathrm{d}}$ \\
& $3 \mathrm{mo}$ & $343 \pm 3^{\mathrm{b}}$ & $0.354 \pm 0.018$ & $+23.1 \pm 2.3^{\mathrm{e}}$ \\
\hline
\end{tabular}

Note: $\mathrm{SD}=$ standard deviation for $\mathrm{n}=3, \mathrm{ND}=$ not determine. Different letters indicate statistically significant differences between group (P value $<0.05)$

\section{DISCUSSION}

The damaged hair occurs from chemical treatments in general and more fracture by mechanical processes for aesthetic purposes, leading to the loss of mechanical strength. The damaged hair results in more brittle fiber and forming a longitudinally split-end, which susceptible to the loss of hair. Although the leave-on product for hair split-end is commercially available, it is notorious for their limitation mainly in low adhesion ability and biocompatibility. These imply to the low efficiency of current hair products for temporarily repair the hair split-end. Hence, this study, NLCs was employed to increase the split-end binding time by its adhesion ability and also improving the biocompatibility of the developed product. Theoretically, the smaller particle size $<200 \mathrm{~nm}$, could provide a large interfacial surface area for particle adhesion to the surface $[14,15]$. Besides the small particle size, the electrostatic charge is a parameter affecting the adhesiveness on hair. Basically, the positively charged particle would offer a high affinity to the negatively charged hair fibers. As a consequence, we aimed to prepare the positively charged NLCs (P-NLCs) and negatively charged NLCs (NLCs) with similar particle size $(<200 \mathrm{~nm})$, to study the influence of electrostatically charged on the hair split-end binding effects.

As previously shown, N-NLCs and P-NLCs were prepared with different solid lipid types to achieve negatively charged particles, $\sim-44 \mathrm{mV}$, and positively charged particles, $32 \mathrm{mV}$, respectively. Both NLCs were successfully prepared with a similar mean particle size using different homogenization cycle. While keeping the same total concentration of lipid, the N-NLCs and P-NLCs were subjected through a high-pressure homogenizer with a homogenization cycle of 5 and 7 respectively. This was due to the hot pre-emulsion of P-NLCs showed a higher viscosity than that of N-NLCs. Thus, the greater homogenization cycle was required to obtain a similar particle size to N-NLCs. Nevertheless, the PNLCs still indicated a slightly lager particle size than N-NLCs. This result could be explained by the different types of lipid used in formulations. In agreement with others studies, the number of the lipid carbon chain has a significant effect on the mean particle size of NLCs [17-19]. As a consequence, P-NLCs prepared with stearyl amine (carbon side chains, C18) resulted in slightly larger particle size than N-NLCs, which contained a stearic acid (carbon side chain, C18) and myristic acid (carbon side chain, C14) at a ratio of 1:1. Nevertheless, both N-NLCs and P-NLCs were found to be suitable for adhesiveness on the hair surface.

As expected, the results suggesting the superiority of both N-NLCs and P-NLCs as they offered better split-end binding time than the cream base, commercial A and B products. The effectiveness of the NLCs could be attributed by two critical factors; the adhesion ability and its film formation coated on the hair, as shown in fig. 4. Firstly, the NLCs could adhere on the hair surface by its small particle in nano-range, and the hydrophobic interaction between its physiological lipid compounds and the cuticle lipid layer (18-Methyl Eicosanoic Acid). Secondly, after adhered on the hair, the NLCs further formed a film by the van der Waals adhesion force which strongly pulled the NLCs particles together [20, 21]. Therefore, the generated NLCs film could pull the split-end fiber back together. In addition, the NLCs films continued coating on the hair surface could retard the split-end reverting after treatment. Apart from the effect of small size and hydrophobic interaction on adhesiveness, we postulated that the positively charged particle could enhance the adhesion on hair and thereby it is essential to compare the influence of NLCs charges in this study. Unexpectedly, both NLCs formulation showed similar split-end binding time, indicating that the positively charged P-NLCs had no extra benefit to enhance the adhesiveness on to negatively charged hair fibers. These results suggest that the developed NLCs with a particle size of $\sim 160 \mathrm{~nm}$ is sufficient to facilitate the particles adhered on the hair, as previously described. Accordingly, both P-NLCs and N-NLCs exhibited the same potential in case of hair split-end binding.

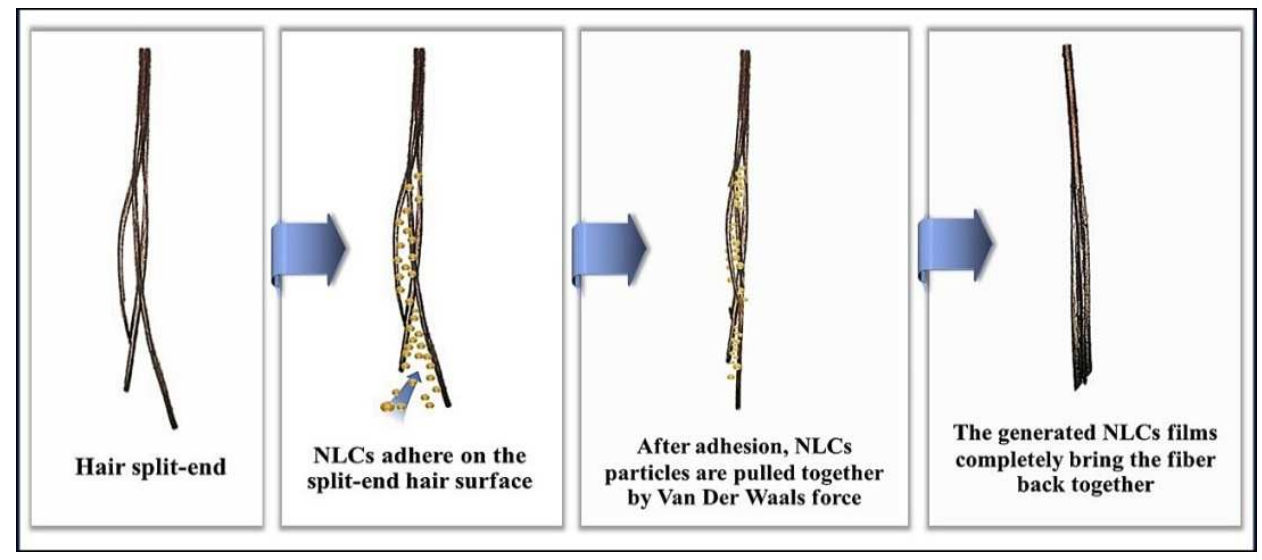

Fig. 4: Proposed mechanisms of NLCs after treated on hair split-end 
The physical stability of N-NLCs and P-NLCs dispersions is also an important issue, due to they possibly show instability in the model cream base. From the results, the N-NLCs showed good physical stability with no evidence of particle aggregation or precipitation when stored at room temperature up to $6 \mathrm{mo}$. On the other hand, after 3-month storage, P-NLCs showed an increased in particle size from 169 to $343 \mathrm{~nm}$, along with a decreased in the zeta potential from $\sim 32 \mathrm{mV}$ to $\sim 23 \mathrm{mV}$. Although an initial high zeta potential of PNLCs of $\sim 30 \mathrm{mV}$ should prevent particle aggregation due to a strong electrostatic repulsion between adjacent of particles, the aggregation was observed as shown by the increased in particle size after 3-mo storage, which leading to phase separation after 6-month storage. The physical instability of P-NLCs can be described by intermolecular ionic interaction between amine group of stearyl amine and phosphate group of phosphatidylcholines (E900) [22, 23].

\section{CONCLUSION}

N-NLCs and P-NLCs were successfully prepared by a high-pressure homogenization technique with a zeta potential of $\sim-44 \mathrm{mV}$ and $\sim 33$ $\mathrm{mV}$ respectively. Additionally, the developed NLCs were prepared with a similar mean size of $\sim 150-160 \mathrm{~nm}$ which aim to improve the adhesion ability. Their nano-sized could facilitate the particles to adhere well on the hair and pull the split end together by forming a film layer. Regardless to the particle surface charge, both NLCs showed similar hair split-end binding time of more than $3 \mathrm{~d}$, suggesting no extra benefit from the positively charged of P-NLCs. In addition, the N-NLCs showed better physical stability than P-NLCs. In conclusion, the developed N-NLCs cream is a novel alternative for hair leave-on product, which could be highly benefits for hair splitend repairing.

\section{ACKNOWLEDGMENT}

This work was supported by Naresuan University and Center of Excellence for Innovation in Chemistry (PERCH-CIC) for the financial support of this work. The authors gratefully acknowledge to the Faculty of Pharmaceutical Sciences, Naresuan University for providing the necessary facilities. Many thanks to Mr. Peter Barton of the Naresuan University Language Centre for his editing assistance and advice on English expression in this document.

\section{AUTHORS CONTRIBUTIONS}

All the author have contributed equally

\section{CONFLICTS OF INTERESTS}

There are no conflicts to declare

\section{REFERENCES}

1. Richena M, Rezende CA. Morphological degradation of human hair cuticle due to simulated sunlight irradiation and washing. J Photochem Photobiol B Biol 2016;161:430-40.

2. Richena $M$, Rezende CA. Effect of photodamage on the outermost cuticle layer of human hair. J Photochem Photobiol B Biol 2015;153:296-304.

3. Swift JA. The mechanics of fracture of human hair. Int J Cosmet Sci 1999;21:227-39.

4. Bhushan B. Nanoscale characterization of human hair and hair conditioners. Prog Mater Sci 2008;53:585-710.
5. Wright M, Szerszen M. Ending the cycle of split ends. Cosmetics Toiletries 2011;126:804-9.

6. Mahajan A. Advancements in polymers used in hair care: a review. Int J Res Cosmet Sci 2016;6:6-16.

7. Nazir H, Wang L, Lian G, Zhu S, Zhang Y, Liu Y, et al. Multilayered silicone oil droplets of narrow size distribution: preparation and improved deposition on hair. Colloids Surfaces B: Biointerfaces 2012;100:42-9.

8. Gavazzoni Dias MF. Hair cosmetics: an overview. Int J Trichol 2015;7:2-15.

9. Dario MF, Baby AR, Velasco MVR. Effects of solar radiation on hair and photoprotection. J Photochem Photobiol B Biol 2015;153:240-6.

10. Duarah S, Pujari K, Durai RD, Narayanan VHB. Nanotechnologybased cosmeceuticals: a review. Int J Appl Pharm 2016;8:8-12.

11. Mehnert W, Mader K. Solid lipid nanoparticles: production, characterization and applications. Adv Drug Delivery Rev 2012;64(Suppl):83-101.

12. Tamjidi F, Shahedi M, Varshosaz J, Nasirpour A. Nanostructured lipid carriers (NLC): a potential delivery system for bioactive food molecules. Innov Food Sci Emerg Technol 2013;19:29-43.

13. Pardeike J, Hommoss A, Müller RH. Lipid nanoparticles (SLN, $\mathrm{NLC}$ ) in cosmetic and pharmaceutical dermal products. Int J Pharm 2009;366:170-84.

14. Müller RH, Radtke M, Wissing SA. Solid lipid nanoparticles (SLN) and nanostructured lipid carriers (NLC) in cosmetic and dermatological preparations. Adv Drug Delivery Rev 2002;54(Suppl):131-55.

15. Müller RH, Petersen RD, Hommoss A, Pardeike J. Nanostructured lipid carriers (NLC) in cosmetic dermal products. Adv Drug Delivery Rev 2007;59:522-30.

16. Mehnert W, Mader K. Solid lipid nanoparticles: production, characterization and applications. Adv Drug Delivery Rev 2012;64(Suppl):83-101.

17. Niamprem P, Srinivas SP, Tiyaboonchai W. Development and characterization of indomethacin-loaded mucoadhesive nanostructured lipid carriers for topical ocular delivery. Int J Appl Pharm 2018;10:91-6.

18. Seyfoddin A, Al-Kassas R. Development of solid lipid nanoparticles and nanostructured lipid carriers for improving ocular delivery of acyclovir. Drug Dev Ind Pharm 2013;39:508-19.

19. Huang ZR, Hua SC, Yang YL, Fang JY. Development and evaluation of lipid nanoparticles for camptothecin delivery: a comparison of solid lipid nanoparticles, nanostructured lipid carriers, and lipid emulsion. Acta Pharmacol Sin 2008;29:1094-102.

20. Katainen J, Paajanen M, Ahtola E, Pore V, Lahtinen J. Adhesion as an interplay between particle size and surface roughness. J Colloid Interface Sci 2006;304:524-9.

21. Ranade MB. Adhesion and removal of fine particles on surfaces. Aerosol Sci Technol 1987;7:161-76.

22. Yang Y, Corona A, Schubert B, Reeder R, Henson MA. The effect of oil type on the aggregation stability of nanostructured lipid carriers. J Colloid Interface Sci 2014;418:261-72.

23. Campbell RB, Balasubramanian SV, Straubinger RM. Phospholipid-cationic lipid interactions: influences on membrane and vesicle properties. Biochim Biophys Acta Biomembr 2001;1512:27-39. 